\title{
U.S. autism prevalence inches upward as racial gaps close
}

\author{
BY PETER HESS
}

2 DECEMBER 2021

\section{Listen to this story:}

https://www.spectrumnews.org/wp-content/uploads/2021/12/audiof2961842-35e0-4eb1-add9-1d1c2a32bfb1-encodings.mp3

Autism prevalence in the United States rose to 1 in 44 children in 2018, up from 1 in 54 in 2016, according to a new study examining autism prevalence among 8-year-olds in 11 states. As in previous years, the uptick is likely due to improvements in identification, diagnosis and referrals to services, rather than a rise in how many children have autism, experts say.

The U.S. Centers for Disease Control and Prevention (CDC) released the report today.

The data are based on health and educational records for 220,281 children, collected as part of the CDC's Autism and Developmental Disabilities Monitoring (ADDM) Network. States in the ADDM Network include Arizona, Arkansas, California, Georgia, Maryland, Minnesota, Missouri, New Jersey, Tennessee, Utah and Wisconsin.

About 4.2 times as many boys as girls were identified as having autism, a sex ratio that is consistent with previous ADDM reports and with other prevalence estimates.

Among children who were 4 years old in 2018, the autism prevalence was 1 in 59 , according to an accompanying report on 220,394 children across the 11 ADDM sites. This figure rose from 2016's estimate of 1 in 64 children. Compared with the older cohort, these children were about 1.5 times as likely to be either diagnosed with autism or identified as needing special education by age 4 , the study found.

!function()\{"use strict";window.addEventListener("message",(function(e)\{if(void $0 !==$.data["datawrapper-height"])\{var t=document.querySelectorAll("iframe");for(var a in 


\section{Spectrum | Autism Research News}

https://www.spectrumnews.org

e.data["datawrapper-height"])for(var $r=0 ; r$ 